NEURODEGENERATIVE DISEASE

\section{Tackling tauopathy}

RNA-binding proteins (RBPs) are implicated in several neurodegenerative diseases. However, it is unclear whether they influence the pathophysiology of neurodegenerative disorders associated with tau pathology (so-called tauopathies), such as Alzheimer disease (AD). Now, Apicco et al. show that reducing the level of the RBP T cell intracellular antigen 1 (TIA1) protects against tau-induced neuronal death, cognitive dysfunction and premature death in mice.

In a previous study, the authors showed in cell culture that expression of a pathological form of tau could

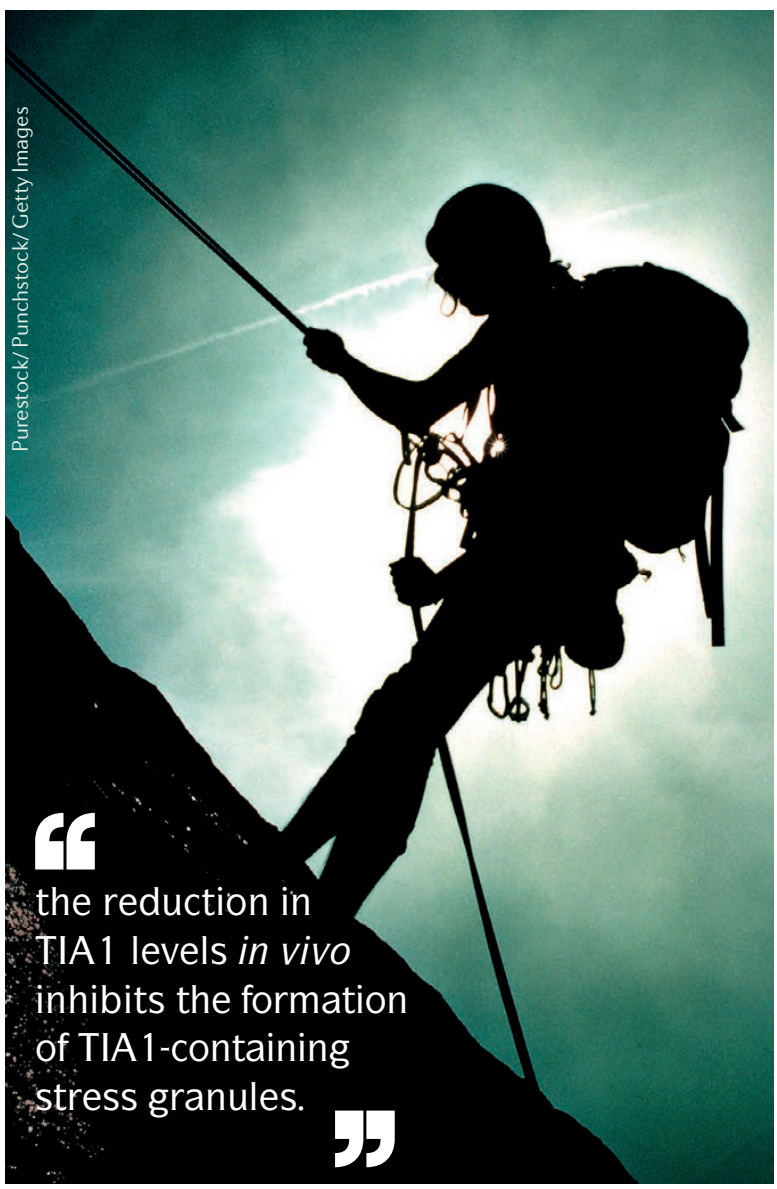

induce TIA1 and other RBPs to aggregate and form stress granules, which are cytoplasmic structures comprising RBPs and RNAs that form under conditions of cellular stress and usually provide a way for cells to temporarily sequester mRNAs that are not required for immediate translation. Interestingly, however, they found that inhibition of stress granule formation - by reducing TIA1 levels - ameliorated signs of tau pathology.

Here, the authors examined whether altering TIA1 levels could influence tau-mediated pathophysiology in vivo. They crossed Tia1 ${ }^{-/-}$mice with PS19 mice, which express a pathological form of human tau (P301S tau), and then crossed selected offspring to generate mice harbouring the P301S tau transgene and either one or both copies of Tia1 (P301S Tial $1^{-/+}$mice and P301S Tial ${ }^{+/+}$ mice, respectively). As expected, P301S Tial ${ }^{-/+}$mice expressed 50\% lower levels of TIA1 than P301S $\mathrm{Tial}^{+/+}$mice.

PS19 mice exhibit a progressive loss of neurons, starting at 6-12 months. By 9 months of age, P301S Tial $^{+/+}$mice showed a reduction in the total number of neurons in the CA3 region of the hippocampus and the lateral entorhinal cortex, and signs of gross cortical atrophy. By contrast, P301S Tia1 ${ }^{-/+}$mice showed no signs of neurodegeneration by this age, indicating that the lower levels of TIA1 protect against P301S tau-induced neural toxicity.

In line with these findings, 6-month-old P301S Tia1 ${ }^{+/+}$mice showed deficits in spatial working memory and recognition memory tasks, and exhibited a shortened lifespan, whereas P301S Tial ${ }^{-/+}$ mice performed similarly to non-transgenic mice in these tasks and they lived, on average, 2 months longer than P301S Tial ${ }^{+/+}$mice.

Hippocampal neurons from P301S Tia1 ${ }^{-1+}$ mice had fewer than half the number of TIA1-positive cytoplasmic granules that P301S $\mathrm{Tial}^{+/+}$mice had and exhibited a similarly sized reduction in the number of granules showing colocalization of TIA1 and a marker of stress granules. Thus, the reduction in TIA1 levels in vivo inhibits the formation of TIA1-containing stress granules.

Tau hyperphosphorylation is a feature of disorders such as AD. Interestingly, by 9 months of age, P301S Tia1 ${ }^{-/+}$mice had a higher level of phosphorylated tau than P301S $\mathrm{Tial}^{+/+}$mice. Moreover, the former showed an increase in the level of aggregated tau, which forms neurofibrillary tangles (NFTs), considered a pathological hallmark of $\mathrm{AD}$. Although the authors found NFTs widely throughout the hippocampus and temporal cortex in P301S Tia1 ${ }^{+/+}$ mice, they also detected similar and higher levels of these structures in the hippocampus and other brain areas, respectively, in P301S Tial $^{-/+}$mice. Thus, in this model, NFT formation does not seem to relate to pathological tau-induced neurotoxicity.

The authors examined whether TIA1 levels influenced the levels of other forms of tau. Strikingly, biochemical experiments revealed that the reduction in TIA1 levels in P301S Tia1 ${ }^{-/+}$mice was associated with a reduction in misfolded and oligomeric species of tau.

Together, these findings indicate that TIA1 may have a central role in conferring tau-induced toxicity, and that this may be related to dysfunction of the cellular stress response pathway and promotion of tau oligomer formation.

Darran Yates

ORIGINAL ARTICLE Apicco, D. J. et al. Reducing the RNA binding protein TIA1 protects against taumediated neurodegeneration in vivo. Nat. Neurosci. http://dx.doi.org/10.1038/s41593-017-0022-z (2017) 